\title{
COMPLEMENTARY SERIES FOR $p$-ADIC GROUPS. I
}

BY

\author{
ALLAN J. SILBERGER ${ }^{1}$
}

\begin{abstract}
Let $\Omega$ be a nonarchimedean local field, $G$ the group of $\Omega$-points of a connected reductive algebraic group defined over $\Omega$. This paper establishes that to each zero of the Plancherel measure of $G$ one can associate complementary series. Our result is the analogue for $p$-adic groups of a similar statement, announced separately by Knapp-Stein and Harish-Chandra, for real groups.
\end{abstract}

Let $\Omega$ be a nonarchimedean local field. Let $G$ be the group of $\Omega$-points of a connected reductive algebraic group $\mathbf{G}$ which is defined over $\Omega$.

In this note we shall prove that one can associate complementary series for $G$ to any zero of the Plancherel measure (cf. Theorem 3.5). Analogous results for real groups have been announced by Harish-Chandra [2a, Theorem 13] and by KnappStein [3, Theorem 4]. Other authors [1], [4], [5] have established the existence of complementary series for $p$-adic groups in special cases.

In further papers of this series we expect to generalize the "standard" facts presented here, and to study the complementary series which may be associated to nontempered unitary representations.

1. Preliminaries. This paper applies and extends Harish-Chandra's theory of induced representations for $p$-adic groups. Although we briefly review notation and terminology, we depend upon [2b] and [6] for essential facts and ideas used here.

We shall refer to algebraic groups defined over $\Omega$ by boldfaced capital letters and to the corresponding groups of $\Omega$-points by the same capital letter in ordinary type. Vector spaces over the complex numbers are denoted by German capital letters.

We fix a minimal $p$-pair $\left(\mathbf{P}_{0}, \mathbf{A}_{0}\right)\left(\mathbf{P}_{0}=\mathbf{M}_{0} \mathbf{N}_{0}\right)$ of $\mathbf{G}$ and an $A_{0}$-good maximal compact subgroup $K$ of $G[6, \S 0.6]$. We assume that all Haar measures on subgroups of $G$ are normalized relative to $K$ (cf. $[6, \S 0.6]$ ). Unless otherwise indicated, all measures on groups will be (left) Haar measures, normalized as above or, if the group is compact, such that the total measure is one.

Let $\mathbf{A}$ be a standard torus of $\mathbf{G}$. We write $\mathscr{P}(\mathbf{A})$ for the set of parabolic subgroups of $\mathbf{G}$ which have $\mathbf{A}$ as a split component. To each $\mathbf{P} \in \mathcal{P}(\mathbf{A})(\mathbf{P}=\mathbf{M N})$ there corresponds a unique "opposite" $\overline{\mathbf{P}} \in \mathscr{P}(\mathbf{A})(\overline{\mathbf{P}}=\mathbf{M} \overline{\mathbf{N}})$ such that $\mathbf{P} \cap \overline{\mathbf{P}}=$ M.

Received by the editors July 2, 1979.

AMS (MOS) subject classifications (1970). Primary 22E50.

Key words and phrases. Complementary series, unitary representations, induced representations.

${ }^{1}$ Research partially supported by the National Science Foundation.

(c) 1980 American Mathematical Society 0002-9947/80/0000-0266/\$03.50 
For any $\mathbf{P} \in \mathcal{P}(\mathbf{A})(\mathbf{P}=\mathbf{M N})$ we write $\delta_{P}$ for the modular function of $\boldsymbol{P}$. Using the Iwasawa decomposition $G=K P$, we set $\delta_{P}(g)=\delta_{P}(p)(g=k p, k \in K, p \in$ $P$ ).

Letting $X(A)$ denote the group of rational characters of the torus $\mathbf{A}$, we write $a^{*}=X(A) \otimes \mathbf{R}, a_{\mathbf{C}}^{*}=a^{*} \otimes \mathbf{C}$, and $a=\operatorname{Hom}(X(A), \mathbf{Z}) \otimes \mathbf{R}$. We identify $X(A)$ with a lattice in $a^{*}$. There is a "log mapping" $H: M \rightarrow \mathfrak{a}$ which sends $M$ to a lattice in a [6, §0.4]. We also set $H_{P}(g)=H(m)$ for $g=k m n(k \in K, m \in M, n \in N)$, $\mathbf{P} \in \mathcal{P}(\mathbf{A})$.

We write $\Sigma(\mathbf{P}, \mathbf{A})$ (respectively, $\left.\Sigma_{r}(\mathbf{P}, \mathbf{A}), \Sigma^{0}(\mathbf{P}, \mathbf{A})\right)$ to denote the set of positive A-roots (respectively, reduced positive A-roots, simple A-roots) corresponding to $P \in \mathcal{P}(\mathbf{A})[6, \S 0.5]$.

Let $\pi$ denote a prime element of $\Omega$. Write "I " to denote the absolute value on $\Omega$ and set $q=\left|\pi^{-1}\right|$. Then there is a unique bilinear form $\langle$,$\rangle on a^{*} \times$ a such that $\log _{a}|\chi(a)|=\langle\chi, H(a)\rangle$ for all $\chi \in X(A)$ and $a \in A$.

For every $\nu=\nu_{1}+\sqrt{-1} \nu_{2} \in a_{C}^{*}\left(\nu_{1}, \nu_{2} \in a^{*}\right)$ set $\bar{\nu}=\nu_{1}-\sqrt{-1} \nu_{2}$ and $\langle\nu, H(m)\rangle=\left\langle\nu_{1}, H(m)\right\rangle+\sqrt{-1}\left\langle\nu_{2}, H(m)\right\rangle(m \in M)$.

We write $\mathcal{E}_{\mathbf{C}}(M)$ (respectively, $\mathcal{E}(M)$ ) for the set of classes of irreducible admissible (respectively, irreducible admissible unitary) representations of $M$. We write $\mathcal{E}_{2}(M)$ for the subset of $\mathcal{E}(M)$ consisting of all discrete series classes.

To each $\nu \in a_{C}^{*}$ (respectively, $a^{*}$ ) there corresponds a quasi-character (respectively, character) $\chi_{\nu}$ of $M$ such that

$$
\chi_{\nu}(m)=q^{\sqrt{-1}\langle\nu, H(m)\rangle} \quad(m \in M) .
$$

For any $\sigma \in \omega \in \mathcal{E}_{\mathbf{C}}(M)$ and $\nu \in \mathrm{a}_{\mathbf{C}}^{*}$ we write $\omega_{\nu}$ for the class of $\sigma \otimes \chi_{\nu}=\sigma_{\nu}$.

Let $W=W(G / A)$ denote the factor group normalizer of $\mathbf{A}$ divided by centralizer of A. For any $\sigma \in \omega \in \mathcal{E}_{\mathbf{C}}(M)$ and representative $y \in G$ of $s \in W$ set $\sigma^{y}\left(y m y^{-1}\right)=\sigma(m)(m \in M)$. The class of the representation $\sigma^{y}$ depends only upon $s$; we denote it $\omega^{s}$. We write $W(\omega)$ for the subgroup of $W$ consisting of all $s$ such that $\omega^{s}=\omega$.

For any admissible representation $\sigma$ of $M$ in a vector space $\mathfrak{U}$ we have the space $\mathfrak{U}^{\sim}$ of smooth linear functionals on $\mathfrak{U}$ and the contragredient representation $\sigma^{\sim}$ of $\boldsymbol{M}$ on $\mathfrak{U}^{\sim}$. There is a nondegenerate bilinear form $\langle$,$\rangle on \mathfrak{U}^{\sim} \times \mathfrak{u}$. Any function on $M$ of the form $x \mapsto\langle\tilde{u}, \sigma(x) u\rangle\left(u \in \mathfrak{U}, \tilde{u^{\sim}} \in \mathfrak{U}\right)$ is called a matrix coefficient of $\sigma$. We write $\mathbb{Q}(\sigma)$ (or $\mathcal{Q}(\omega)$, if $\omega$ denotes the class of $\sigma$ ) for the vector space spanned by the set of matrix coefficients of $\sigma$.

Given an admissible representation $\sigma$ of $M$ in a vector space $\mathfrak{U}$, we write $\operatorname{End}^{0}(\mathfrak{U})$ for the space of all double $K$-finite endomorphisms of $\mathfrak{u}[6, \S 1.11]$. For any pair

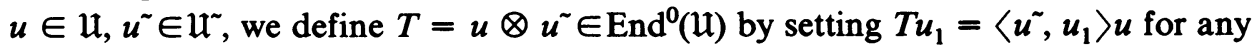
$u_{1} \in \mathfrak{U}$. Extending by linearity, we obtain a canonical isomorphism of $\mathfrak{U} \otimes \mathfrak{U}^{\sim}$ onto $\operatorname{End}^{0}(\mathfrak{u})$.

Since every element of $\operatorname{End}^{0}(\mathfrak{U})$ has finite rank, and therefore a trace, it follows that, whenever $\mathfrak{U}$ has the structure of a prehilbert space, End ${ }^{0}(\mathfrak{U})$ does too: $\|T\|^{2}=\operatorname{trace}\left(T T^{*}\right)$ for every $T \in \operatorname{End}^{0}(\mathfrak{H})\left(T^{*}\right.$ denotes the adjoint of $\left.T\right)$.

Let $C^{\infty}(M)$ denote the space of locally constant complex-valued functions on $M, C_{c}^{\infty}(M)$ the subspace consisting of the compactly supported functions. Then 
$C_{c}^{\infty}(M)$ is a convolution algebra on the unimodular group $M$. For any admissible representation $\sigma$ of $M$, setting $\sigma(f)=\int_{M} f(m) \sigma(m) d m\left(f \in C_{c}^{\infty}(M)\right)$ defines a homomorphism of the algebra $C_{c}^{\infty}(M)$ into $\operatorname{End}^{0}(\mathfrak{U})$.

Let $(\mathfrak{B}, \tau)$ be a smooth unitary double representation of $K$ which satisfies associativity conditions $[6, \S 1.12]$. Let $\tau_{M}$ denote the restriction of $\tau$ to $K \cap M$. Given $\mathbf{P}_{1}, \mathbf{P}_{2} \in \mathcal{P}(\mathbf{A})\left(\mathbf{P}_{i}=\mathbf{M N}_{i}, i=1,2\right)$, we write $\mathfrak{B}_{P_{1} \mid P_{2}}$ for the set of all $v \in \mathfrak{V}$ such that $\tau\left(n_{1}\right) v \tau\left(n_{2}\right)=v\left(n_{i} \in N_{i} \cap K, i=1,2\right)$. Then $\mathfrak{P}_{P_{1} \mid P_{2}}$ is $\tau_{M}$-stable. We write $\tau_{P_{1} \mid P_{2}}$ for the double representation of $K \cap M$ on $\mathfrak{B}_{P_{1} \mid P_{2}}$.

We write $C(G, \tau)$ for the space of all $\mathfrak{B}$-valued functions $f$ on $G$ such that $f\left(k_{1} g k_{2}\right)=\tau\left(k_{1}\right) f(g) \tau\left(k_{2}\right) \quad\left(k_{1}, k_{2} \in K, g \in G\right), C_{c}(G, \tau)$ for the subspace of $C(G, \tau)$ consisting of the compactly supported functions. Since $\mathfrak{B}$ is a selfadjoint algebra, $C_{c}(G, \tau)$ has a natural structure of selfadjoint convolution algebra on $G$. Writing $v^{*}$ for the adjoint of $v \in \mathfrak{B}$, we set $\alpha^{\sim}(x)=\alpha\left(x^{-1}\right)^{*}$ and call $\alpha^{\sim}$ the adjoint of $\alpha \in C_{c}(G, \tau)$. Of course, the convolution products $\alpha * f$ and $f * \alpha$ exist for any $f \in C(G, \tau)$ and $\alpha \in C_{c}(G, \tau)$.

We also have the spaces $C\left(M, \tau_{M}\right)$ and $C_{c}\left(M, \tau_{M}\right)$ associated to $M$ and $\left(\mathfrak{B}, \tau_{M}\right)$ with the same properties. We write $C\left(M, \tau_{P_{1} \mid P_{2}}\right)$ for the subspace of $C\left(M, \tau_{M}\right)$ associated to $\left(\mathfrak{B}_{P_{1} \mid P_{2}}, \tau_{P_{1} \mid P_{2}}\right)\left(\mathbf{P}_{1}, \mathbf{P}_{2} \in \mathscr{P}(\mathbf{A})\right)$. For any $\alpha \in C_{c}(G, \tau)$ and $\mathbf{P} \in \mathscr{P}(\mathbf{A})$ $(\mathbf{P}=\mathbf{M N})$ set

$$
\alpha^{P}(m)=\delta_{P}^{1 / 2}(m) \int_{N} \alpha(m n) d m \quad(m \in M) .
$$

Then $\alpha \mapsto \alpha^{P}$ is an algebra homomorphism of $C_{c}(G, \tau)$ to $C_{c}\left(M, \tau_{P \mid P}\right)[6$, Lemma 2.7.5]. We also have $\left(\alpha^{\sim}\right)^{P}=\left(\alpha^{P}\right)^{\sim}[6$, Lemma 2.7.9].

For any admissible representation $\sigma$ of $M$ and $\mathbf{P}_{1}, \mathbf{P}_{2} \in \mathscr{P}(\mathbf{A})$ we write

$$
\mathbb{Q}\left(\sigma, \tau_{P_{1} \mid P_{2}}\right)=\left(\mathbb{Q}(\sigma) \otimes \mathfrak{B}_{P_{1} \mid P_{2}}\right) \cap C\left(M, \tau_{P_{1} \mid P_{2}}\right) .
$$

The space $\mathcal{Q}\left(\sigma, \tau_{P_{1} \mid P_{2}}\right)$ is a left module for $C_{c}\left(M, \tau_{P_{1} \mid P_{1}}\right)$ and a right module for $C_{c}\left(M, \tau_{P_{2} \mid P_{2}}\right)$.

We shall need the double representation $\left(\mathfrak{B}^{0}, \tau^{9}\right)$, where $\mathfrak{B}^{0}=C^{\infty}(K \times K)$ and, for any $v \in \mathfrak{B}^{0}$,

$$
\tau^{0}\left(k_{1}\right) v\left(l_{1}: l_{2}\right) \tau^{0}\left(k_{2}\right)=v\left(l_{1} k_{1}: k_{2} l_{2}\right) \quad\left(k_{i}, l_{i} \in K, i=1,2\right) .
$$

We set $v^{*}\left(k_{1}: k_{2}\right)=\bar{v}\left(k_{2}^{-1}: k_{1}^{-1}\right)\left(k_{1}, k_{2} \in K, v \in \mathfrak{B}^{9}\right)$ and

$$
\begin{gathered}
v_{1} \cdot v_{2}\left(k_{1}: k_{2}\right)=\int_{K} v_{1}\left(k_{1}: l\right) v_{2}\left(l^{-1}: k_{2}\right) d l \quad\left(k_{i} \in K, v_{i} \in \mathfrak{B}^{0}, i=1,2\right), \\
\|v\|_{\mathfrak{B}^{0}}^{2}=\int_{K \times K}\left|v\left(k_{1}: k_{2}\right)\right|^{2} d k_{1} d k_{2} .
\end{gathered}
$$

For any $f \in C^{\infty}(G)$ we set $\mathbf{f}\left(k_{1}: g: k_{2}\right)=f\left(k_{1} g k_{2}\right)\left(k_{1}, k_{2} \in K, g \in G\right)$. The mapping $j: f \mapsto f$ is a bijection of $C^{\infty}(G)$ onto $C\left(G, \tau^{0}\right), C_{c}^{\infty}(G)$ onto $C_{c}\left(G, \tau^{0}\right)$, and $\mathcal{Q}(\pi)$ onto $\mathcal{Q}\left(\pi, \tau^{9}\right)$ for any admissible representation $\pi$ of $G$. We have $j\left(f_{1} * f_{2}\right)=$ $f_{1} * f_{2}$ whenever either product exists.

Let $\sigma$ be an admissible representation of $M$ in a vector space $\mathfrak{U}$. Regarding $\sigma$ as a representation of $P, \mathbf{P} \in \mathscr{P}(\mathbf{A})$, we write $I(P, \sigma)$ for the representation of $G$ by 
right translations in the space $\mathscr{G}(P)=\mathscr{B}(P, \sigma)$ which consists of all smooth $\mathcal{U}$-valued functions $h(g)$ on $G$ such that $h(p g)=\delta_{P}^{1 / 2}(p) \sigma(p) h(g),(p \in P, g \in G)$. Write $I(P, \omega)$ for the class of $I(P, \sigma)$.

Let $\sigma \in \omega \in \mathcal{E}_{\mathbf{C}}(M)$ and let $(\mathfrak{B}, \tau)$ be a double representation of $K$ as above. Let $\mathbf{P} \in \mathcal{P}(\mathbf{A})(\mathbf{P}=\mathbf{M N})$. For any $\psi \in \mathbb{Q}\left(\omega, \tau_{P \mid P}\right)$ and $x=k m n \in G(k \in K, m \in M$, $n \in N)$, we set $\psi(x)=\tau(k) \psi(m)$. For any $\nu \in a_{\mathbf{C}}^{*}$ and $g \in G$ Harish-Chandra defines the "Eisenstein integral"

$$
\begin{aligned}
E(P: \psi: \nu: g) & =E\left(P: \psi \chi_{\nu}: g\right) \\
& =\int_{G} \psi(g k) \tau\left(k^{-1}\right) q^{\sqrt{-1}\left\langle\nu, H_{P}(x k)\right\rangle} \delta_{P}^{-1 / 2}(x k) d k .
\end{aligned}
$$

Then $E(P: \psi: \nu) \in \mathbb{Q}\left(I\left(P, \omega_{\nu}\right), \tau\right)[6$, Corollary 5.2.2.5].

If $\omega \in \mathcal{E}_{2}(M)$, then $I\left(P, \sigma_{\nu}\right)$ is tempered for all $\nu \in a^{*}$. For any $\mathbf{P}_{1}, \mathbf{P}_{2} \in \mathcal{P}(\mathbf{A})$ we have the weak constant term

$$
{ }_{w} E_{P_{2}}\left(P_{1}: \psi: \nu\right)=\sum_{s \in W} c_{P_{2} \mid P_{1}}(s: \omega: \nu) \psi \chi_{s \nu}
$$

where the mapping

$$
c_{P_{2} \mid P_{1}}(s: \omega: \nu): \mathbb{Q}\left(\omega, \tau_{P_{1} \mid P_{1}}\right) \rightarrow \mathbb{Q}\left(\omega^{s}, \tau_{P_{2} \mid \bar{P}_{2}}\right)
$$

is, for every $s \in W$, a meromorphic function on $a_{\mathbf{C}}^{*}$ which is almost everywhere bijective. With respect to the norm $\|\psi\|^{2}=\int_{M / A}\|\psi(x)\|_{\mathfrak{B}}^{2} d x^{*}, c_{P_{2} \mid P_{1}}(s: \omega: \nu)$ has an adjoint $c_{P_{2} \mid P_{1}}(s: \omega: \nu)^{*}$. Moreover, there exists a meromorphic scalar-valued function $\mu(\omega: \nu)\left(\nu \in a_{C}^{*}\right)$, which is holomorphic and assumes nonnegative real values on $a^{*}$, such that $c_{P_{2} \mid P_{1}}(s: \omega: \bar{\nu})^{*} \mu(\omega: \nu)$ and $c_{P_{2} \mid P_{1}}(s: \omega: \nu)$ are inverses of one another wherever both are defined.

For any $\mathbf{P} \in \mathcal{P}(\mathbf{A}), \nu \in a_{\mathbf{C}}^{*}$, and $\phi \in \mathbb{Q}\left(\omega, \tau_{P \mid \bar{P}}\right)$ Harish-Chandra also defines (as a meromorphic function)

$$
E^{0}(P: \phi: \nu)=E\left(P: c_{P \mid P}(1: \omega: \nu)^{-1} \phi: \nu\right)
$$

and operators

$$
c_{P_{2} \mid P_{1}}^{0}(s: \omega: \nu)=c_{P_{P_{2} \mid P_{1}}}(s: \omega: \nu) c_{P_{1} \mid P_{1}}(1: \omega: \nu)^{-1},
$$

$\left(s \in W, \mathbf{P}_{1}, \mathbf{P}_{2} \in \mathcal{P}(\mathbf{A})\right)$ such that

$$
{ }_{w} E_{P_{2} \mid P_{1}}^{0}\left(P_{1}: \phi: \nu\right)=\sum_{s \in W} c_{P_{2} \mid P_{1}}^{0}(s: \omega: \nu) \phi \chi_{s \nu}
$$

The $c^{0}$-functions are holomorphic and unitary on $a^{*}([2 b, \S 12]$ or $[6, \S 5.3 .5])$. The various functional equations satisfied by the $c$-functions and $c^{0}$-functions are given in [2b, \$12], assuming $\omega$ supercuspidal, and proved for all $\omega \in \mathcal{E}_{2}(M)$ in $[6, \S 5.3 .5]$.

2. Two lemmas relating the $c$-functions to intertwining operators. Let $A$ be a standard torus of $\mathbf{G}, \mathbf{M}$ the centralizer of $\mathbf{A}$. Let $\sigma \in \omega \in \mathcal{E}_{2}(M)$. Let $(\mathfrak{B}, \tau)$ denote a smooth unitary double representation of $K$ which satisfies the conditions of [6, $\S 1.12]$. 
Lemma 2.1. Let $\mathbf{P}_{1}, \mathbf{P}_{2} \in \mathcal{P}(\mathbf{A})$ and $s \in W$. Let $\alpha, \beta \in C_{c}(G, \tau)$ and $\psi \in$ $\mathcal{Q}\left(\omega, \tau_{P_{1} \mid P_{1}}\right)$. Then

$$
\alpha^{P_{2}}\left(c_{P_{2} \mid P_{1}}(s: \omega: \nu) \psi\right) \chi_{s \nu} * \beta^{\bar{P}_{2}}=\chi_{s \nu} c_{P_{2} \mid P_{1}}(s: \omega: \nu)\left(\chi_{-\nu}\left(\alpha^{P_{1}} * \psi \chi_{v} * \beta^{P_{1}}\right)\right)
$$

for all $\nu \in a_{C}^{*}$ such that the c-function is defined.

Proof. It suffices to check this relation on an $h$-dense subset of $a_{c}^{*}$, i.e., take $\omega_{\nu}$ such that $W\left(\omega_{\nu}\right)$ is trivial. The general statement follows via analytic continuation.

Using [6, Lemmas 5.2.2.1-5.2.2.3], we have

$$
\alpha * E\left(P_{1}: \psi: \nu\right) * \beta=E\left(P_{1}: \alpha^{P_{1}} * \psi \chi_{\nu} * \beta^{P_{1}}\right)
$$

Therefore,

$$
\left(\alpha * E\left(P_{1}: \psi: \nu\right) * \beta\right)_{P_{2}, s}=\chi_{s \nu} c_{P_{2} \mid P_{1}}(s: \omega: \nu)\left(\chi_{-\nu}\left(\alpha^{P_{1}} * \psi \chi_{\nu} * \beta^{P_{1}}\right)\right) .
$$

On the other hand, by [6, Lemma 2.7.6 and §3.1],

$$
\left(\alpha * E\left(P_{1}: \psi: \nu\right) * \beta\right)_{P_{2}, s}=\alpha_{M}^{P_{2}} * E_{P_{2}, s}\left(P_{1}: \psi: \nu\right) * \beta_{M}^{\bar{P}_{2}}
$$

The lemma follows by substitution.

Let $\sigma$ act in a prehilbert space $\mathfrak{U}$. Set $\sigma^{0}=\sigma \mid K \cap M$, and assume that $\sigma^{0}$ is unitary. Let $I^{0}$ denote the unitary admissible representation of $K$ by right translations in the prehilbert space $\mathfrak{S}$ consisting of all locally constant $\mathfrak{U}$-valued functions on $K$ such that $h(m k)=\sigma^{0}(m) h(k)(m \in M \cap K, k \in K)$. For any $\mathbf{P} \in \mathcal{P}(\mathbf{A})$ $(\mathbf{P}=\mathbf{M N})$ let $\mathscr{G}(P)$ denote the subspace of $\mathscr{S}$ consisting of all left $(N \cap K)$ invariant functions, let $F(P)$ denote the projection of $\mathscr{S}$ on $\$(P)$ and let $I^{0}(P)$ denote the representation $I^{0}$ on $\mathscr{Q}(P)$. Let $\mathcal{T}$ denote End ${ }^{0}(\mathscr{Q})$; for any $\mathbf{P}_{1}, \mathbf{P}_{2} \in$ $\mathscr{P}(\mathbf{A})$ set $\mathcal{T}\left(P_{1} \mid P_{2}\right)=F\left(P_{1}\right) \mathcal{T} F\left(P_{2}\right)$.

Since $I^{0}(P)$ is equivalent to $I\left(P, \sigma_{\nu}\right) \mid K$ for any $\nu \in a_{C}^{*}$ and $\mathbf{P} \in \mathscr{P}(\mathbf{A})$, we may regard $I\left(P, \sigma_{\nu}\right)$ as acting in $\mathscr{B}(P)$. For each $\nu \in a_{C}^{*}$ we have the algebra homomorphism $\alpha \mapsto I\left(P, \sigma_{v}\right)(\alpha)$ from $C_{c}^{\infty}(G)$ to $\mathscr{T}(P \mid P)$.

Since the algebra $\mathcal{T}$ is isomorphic to $\mathscr{B} \otimes \mathfrak{S}^{\sim}$, it follows that, for every $T \in \mathcal{T}$, there is a locally constant kernel $\kappa_{T}: K \times K \rightarrow \operatorname{End}^{0}(\mathfrak{U})$ such that

$$
T h(k)=\int_{K} \kappa_{T}(k: l) h\left(l^{-1}\right) d l \quad(h \in \mathfrak{Z}) .
$$

We set

$$
\psi_{T}\left(k_{1}: m: k_{2}\right)=\operatorname{trace}\left(\sigma(m) \kappa_{T}\left(k_{2}: k_{1}\right)\right) \quad\left(m \in M, k_{1}, k_{2} \in K\right)
$$

for every $T \in \mathcal{T}$. The mapping $T \mapsto \psi_{T}$ defines a bijection of $\mathcal{T}$ on $\mathcal{Q}\left(\omega, \tau_{M}^{0}\right)$ [6, Lemma 5.2.1.3]. For any $P_{1}, P_{2} \in \mathcal{P}(\mathbf{A})$ and $T \in \mathcal{T}\left(P_{2} \mid P_{1}\right), \psi_{T} \in \mathcal{Q}\left(\omega, \tau_{P_{1} \mid P_{2}}^{0}\right)$. Given $\alpha \in C_{c}^{\infty}(G)$ and $T \in \mathcal{T}\left(P_{2} \mid P_{1}\right)$, we have, by [6, Lemma 5.2.4.1],

$$
\alpha^{P_{1}} * \psi_{T}=\psi_{T I\left(P_{1}, \sigma\right)\left(\alpha^{\prime}\right)} \text { and } \psi_{T} * \alpha^{P_{2}}=\psi_{I\left(P_{2}, \sigma\right)\left(\alpha^{\prime}\right) T},
$$

$\left(\alpha^{\prime}(x)=\alpha\left(x^{-1}\right)\right.$ for all $\left.x \in G\right)$. Since $\omega \in \mathcal{E}_{2}(M), \mathcal{Q}\left(\omega, \tau_{M}^{0}\right)$ is both an algebra and a prehilbert space. Sending $T \mapsto \psi_{T}$ induces both an anti-isomorphism of algebras and a unitary isomorphism of the prehilbert spaces (cf. [6, Lemma 5.2.4.1]). 
Explicitly, we have $\|T\|^{2}=d(\omega)^{-1} \int_{M / A}\left\|\psi_{T}(x)\right\|_{\mathfrak{F}^{\circ}}^{2} d x^{*}$ for any $T \in \mathcal{T}(d(\omega)$ denotes the formal degree of $\omega$ relative to the normalized Haar measures).

Now fix $\mathbf{P}_{1}, \mathbf{P}_{2} \in \mathcal{P}(\mathbf{A})$ and $s \in W(\omega)$. Define $c(s: \nu): \mathcal{T}\left(P_{1} \mid P_{1}\right) \rightarrow \mathcal{T}\left(\bar{P}_{2} \mid P_{2}\right)$ by setting $c_{P_{2} \mid P_{1}}(s: \omega: \nu) \psi_{T}=\psi_{c(s: \nu)(T)}$ for any $T \in \mathcal{T}\left(P_{1} \mid P_{1}\right)$.

Lemma 2.2. Let $\alpha, \beta \in C_{c}^{\infty}(G)$ and $T \in \mathcal{T}\left(P_{1} \mid P_{1}\right)$. Then

$$
I\left(\bar{P}_{2}, \sigma_{s v}\right)(\beta) c(s: \nu)(T) I\left(P_{2}, \sigma_{s v}\right)(\alpha)=c(s: \nu)\left(I\left(P_{1}, \sigma_{v}\right)(\beta) T I\left(P_{1}, \sigma_{v}\right)(\alpha)\right) \text {. }
$$

Proof. For any $T \in \mathcal{T}\left(P_{1} \mid P_{1}\right)$ we have

$$
\alpha^{P_{1}} * \psi_{T} \chi_{\nu} * \beta^{P_{1}}=\chi_{v} \psi_{I\left(P_{1}, \sigma_{v}\right)\left(\beta^{\prime}\right) T I\left(P_{1}, \sigma_{v}\right)\left(\alpha^{\prime}\right)}
$$

and

$$
\alpha^{P_{2}} * c_{P_{2} \mid P_{1}}(s: \omega: \nu) \psi_{T} \chi_{s v} * \beta^{\bar{P}_{2}}=\chi_{s \nu} \psi_{I}\left(\bar{P}_{2}, \sigma_{s}\right)\left(\beta^{\prime}\right) c(s: \nu)(T) I\left(P_{2}, \sigma_{s}\right)\left(\alpha^{\prime}\right) \text {. }
$$

Combining these relations with Lemma 2.1 completes the proof of the present lemma.

3. The existence of complementary series. Let $(P, A)(P=M N)$ be a semistandard $p$-pair of $\mathbf{G},(\mathfrak{B}, \tau)$ as before. Let $\sigma \in \omega \in \mathcal{E}_{2}(M)$. For any $s \in W(G / A)$ define $a^{*}(s)=\left\{\nu \in a^{*} \mid s \nu=-\nu\right\}$.

LEMMA 3.1. Let $s \in W(\omega)$. The function $\mu(\omega: \nu)$ is real valued for all $\nu$ $\in \sqrt{-1} a^{*}(s)$.

Proof. By [6, Theorem 5.3.5.2] (cf. [2b, Theorem 20]) the identity on $\mathcal{Q}\left(\omega, \tau_{P \mid \bar{P}}\right)$ equals $\mu(\omega: \nu) c_{P \mid P}(1: \omega: \nu) c_{P \mid P}(1: \omega: \bar{\nu})^{*}$ for all $\nu \in a_{C}^{*}$. Taking adjoints, we find that

$$
\mu(\omega: \nu) c_{P \mid P}(1: \omega: \nu) c_{P \mid P}(1: \omega: \bar{\nu})^{*}=\bar{\mu}(\omega: \nu) c_{P \mid P}(1: \omega: \bar{\nu}) c_{P \mid P}(1: \omega: \nu)^{*},
$$

which implies that $\mu(\omega: \bar{\nu})=\bar{\mu}(\omega: \nu)$ for all $\nu \in a_{C}^{*}$ where these functions are defined. If $\nu \in \sqrt{-1} a^{*}(s)$, then

$$
\mu(\omega: \nu)=\mu\left(\omega^{s}: s \nu\right)=\mu(\omega:-\nu)=\mu(\omega: \bar{\nu}) .
$$

LeMma 3.2. Let $s \in W(\omega), s^{2}=1$, and $\nu \in \sqrt{-1} \mathrm{a}^{*}(s)$. Then $c_{P \mid P}^{0}(s: \omega: \nu)$ is a selfadjoint operator on the prehilbert space $\mathcal{Q}\left(\omega, \tau_{P \mid \bar{P}}\right)$ (cf. [2a, Lemma 19]).

Proof. Since $s^{2}=1$ and $\nu \in \sqrt{-1} a^{*}(s)$, the functional equations for the $c^{0}$ function imply that $c_{P \mid P}^{0}(s: \omega: \nu)=c_{P \mid P}^{0}(s: \omega:-\nu)^{-1}$. Therefore,

$$
\begin{aligned}
c_{P \mid P}^{0}(s: \omega: \nu) & =c_{P \mid P}(1: \omega:-\nu) c_{P \mid P}(s: \omega:-\nu)^{-1} \\
& =\left(\mu(\omega: \bar{\nu}) c_{P \mid P}(1: \omega: \bar{\nu})\right)\left(\mu(\omega: \bar{\nu}) c_{P \mid P}(s: \omega: \bar{\nu})\right)^{-1} \\
& =\left(c_{P \mid P}(1: \omega: \nu)^{*}\right)^{-1} c_{P \mid P}(s: \omega: \nu)^{*} \\
& =\left(c_{P \mid P}(s: \omega: \nu) c_{P \mid P}(1: \omega: \nu)^{-1}\right)^{*}=c_{P \mid P}^{0}(s: \omega: \nu)^{*} .
\end{aligned}
$$

Now let $s \in W(\omega)$ be such that $s^{2}=1$. Assume that there exists a sequence of reduced A-roots $\alpha_{1}, \ldots, \alpha_{r}$ such that the reflections $s_{i}=s_{\alpha_{i}}(i=1, \ldots, r)$ with respect to the hyperplanes $\alpha_{i}^{-1}(0) \subset a$ lie in $W(\omega)$. Assume also that the factors 
$\mu_{\alpha_{i}}(\omega: \nu)$ of $\mu(\omega: \nu)$ have zeroes at $\nu=0$ for all $i=1, \ldots, r$. In this case, if $s=s_{r} \ldots s_{1}$, we call $s \omega$-complementary of length $r$.

For the remainder of the paper let $s \in W(\omega)$ be $\omega$-complementary of length $r \geqslant 1$. Set $C(\nu)=(-1)^{r} c_{P \mid P}^{0}(s: \omega: \nu)\left(\nu \in a_{C}^{*}\right)$.

LEMMA 3.3. $C(0)$ is the identity on $\mathcal{Q}\left(\omega, \tau_{P \mid \bar{P}}\right)$.

Proof. Let $s=s_{r} \ldots s_{1}$ be as above. By [6, Theorem 5.3.5.3.(2)], [2b, Theorem 21(4)] we have

$$
\begin{aligned}
c_{P \mid P}^{0}(s: \omega: \nu)=c_{P \mid P}^{0}\left(s_{r}: \omega: s_{r-1} \cdots s_{1} \nu\right) c_{P \mid P}^{0}\left(s_{r-1}: \omega: s_{r-2}\right. & \left.\cdots s_{1} \nu\right) \\
& \cdots c_{P \mid P}^{0}\left(s_{1}: \omega: \nu\right) .
\end{aligned}
$$

It is sufficient to show that, for each $i, c_{P \mid P}^{0}\left(s_{i}: \omega: 0\right)$ is minus the identity on $\mathcal{Q}\left(\omega, \tau_{P \mid \bar{P}}\right)$. For each $i$ there exists $\mathbf{Q}_{i} \in \mathcal{P}(\mathbf{A})$ such that $\alpha_{i} \in \Sigma^{0}\left(\mathbf{Q}_{i}, \mathbf{A}\right)$. Since

$$
c_{P \mid P}^{0}\left(s_{i}: \omega: 0\right)=c_{P \mid Q_{i}}^{0}(1: \omega: 0) c_{Q_{i} \mid Q_{i}}^{0}\left(s_{i}: \omega: 0\right) c_{Q_{i} \mid P}^{0}(1: \omega: 0)
$$

and

$$
c_{P \mid Q_{i}}^{0}(1: \omega: 0) c_{Q_{i} \mid P}^{0}(1: \omega: 0)=1_{Q\left(\omega, \tau_{P \mid}\right)},
$$

it is enough to show that $c_{Q_{i} \mid Q_{i}}^{0}\left(s_{i}: \omega: 0\right)$ is minus the identity on $\mathcal{Q}\left(\omega, \tau_{Q_{2} \mid \bar{Q}_{2}}\right)$.

Let $\mathbf{A}_{i}$ be the largest subtorus of $\mathbf{A}$ lying in the kernel of the root character $\alpha_{i}$ and let $\mathbf{M}_{i}$ denote the centralizer of $\mathbf{A}_{i}$. Then $\mathbf{M}_{i}$ is a connected reductive group and $\left({ }^{*} \mathbf{P}_{i}, \mathbf{A}\right)$, where ${ }^{*} \mathbf{P}_{i}=\mathbf{Q}_{i} \cap \mathbf{M}_{i}$, is a maximal $p$-pair of $\mathbf{M}_{i}$. By [6, Theorem 5.3.5.3(5)], [2b, Theorem 18],

$$
c_{Q_{1} \mid Q_{i}}^{0}\left(s_{i}: \omega: \nu\right)=\left.c_{{ }_{P_{i} \mid}^{0} P_{i}}^{0}\left(s_{i}: \omega: \nu\right)\right|_{\mathbb{Q}\left(\omega, \tau_{Q_{1} \mid \bar{Q}_{i}}\right)}
$$

for all $\nu \in a_{C}^{*}$. It is enough to consider the case in which $s$ is $\omega$-complementary of length one and $(\mathbf{P}, \mathbf{A})$ is a maximal $p$-pair of $\mathbf{G}$.

To check this case we recall that our hypotheses imply that, for any $\phi \in$ $\mathcal{Q}\left(\omega, \tau_{P \mid \bar{P}}\right)$, we have

$$
{ }_{w} E_{P}^{0}(P: \phi: \nu)=\phi \chi_{\nu}+c_{P \mid P}^{0}(s: \omega: \nu) \phi \chi_{s \nu},
$$

which is a holomorphic function in some neighborhood of $\nu=0$ in $a_{C}^{*}$ [6, Corollary 5.3.3.5]. It is enough to show that ${ }_{w} E_{P}^{0}(P: \phi: 0)=0$.

By the Maass-Selberg relations ([2b, Theorem 15] or [6, Theorem 5.2.4.4]) and the hypothesis $\mu(\omega: 0)=0$,

$$
E^{0}(P: \phi: 0)=\lim _{\nu \rightarrow 0} E^{0}(P: \phi: \nu)=\lim _{\nu \rightarrow 0} E\left(P: c_{P \mid P}(1: \omega: \nu)^{-1} \phi: \nu\right)=0 .
$$

(i.e., $\mu(\omega: \nu)^{-1}\left\|c_{P \mid P}(1: \omega: \nu)^{-1} \phi\right\|^{2}$ independent of $\nu \in a^{*}$ (Maass-Selberg) and $\mu(\omega: 0)=0$ imply that $\lim _{\nu \rightarrow 0} c_{P \mid P}(1: \omega: \nu)^{-1} \phi=0$. Clearly,

$$
\left.\lim _{\nu \rightarrow 0} E\left(P: c_{P \mid P}(1: \omega: \nu)^{-1} \phi: \nu\right)=E\left(P: \lim _{\nu \rightarrow 0} c_{P \mid P}(1: \omega: \nu)^{-1} \phi: 0\right)=0 .\right)
$$

Therefore, since

$$
E^{0}(P: \phi: \nu: m a)=\delta_{P}^{-1 / 2}(m a) E_{P}^{0}(P: \phi: \nu: m a)
$$


for $m \in M$ and $a=a(m)$ suitably chosen but independent of $\nu$, we may conclude, on account of the $A$-finiteness of $E_{P}^{0}$, that $\lim _{\nu \rightarrow 0} E_{P}^{0}(P: \phi: \nu)=0$, too. This implies easily, and we omit the explicit justification, that $\lim _{\nu \rightarrow 0} E_{P}^{0}(P: \phi: \nu)=0$, whence the lemma.

LEMMA 3.4. There exists a neighborhood $U$ of zero in $\sqrt{-1} a^{*}(s)$ such that, for all $\nu \in U, C(\nu)$ is positive definite.

Proof. Since $C(\nu)$ is an analytic function near $\nu=0$, its eigenvalues vary continuously. Since $C(0)=I_{\mathscr{Q}\left(\omega, \tau_{P \mid \bar{P}}\right)}$ and since $C(\nu)$ is hermitian for all $\nu$ $\in \sqrt{-1} a^{*}(s)$, it is enough to show that there exists a neighborhood $U_{1}$ of $\nu=0$ in $a_{C}^{*}$ such that $C(\nu)$ is bijective for all $\nu \in U_{1}$. Since the eigenvalues of $C(\nu)$ are real for all $\nu \in \sqrt{-1} a^{*}(s)$, if $C(\nu)$ is bijective for all $\nu \in U_{1}$, then the eigenvalues of $C(\nu)$ are positive for all $\nu \in U=U_{1} \cap \sqrt{-1} a^{*}(s)$.

Because of the functional equation

$$
c_{P \mid P}^{0}(s: \omega: \nu)=c_{P \mid P}^{0}\left(s_{r}: \omega: s_{r-1} \cdots s_{1} \nu\right) \cdots c_{P \mid P}^{0}\left(s_{1}: \omega: \nu\right),
$$

it is enough to show that $C(\nu)$ is bijective in a neighborhood of zero when $r=1$. Indeed, by the same reasoning as in the proof of Lemma 3.3, it is even enough to consider the case in which $(\mathbf{P}, \mathbf{A})$ is a maximal $p$-pair.

Assume, then, that $(\mathbf{P}, \mathbf{A})$ is maximal and that $W(\omega)=\{1, s\}$. Since the weak constant term is analytic in a neighborhood $U_{1}$ of 0 in $a_{C}^{*}$ the $c$-functions $c_{P \mid P}(1: \omega: \nu)$ and $c_{P \mid P}(s: \omega: \nu)$ and the adjoints $c_{P \mid P}(1: \omega: \bar{\nu})^{*}$ and $c_{P \mid P}(s: \omega: \bar{\nu})^{*}$ are analytic functions for all $\nu \in U_{1}$ such that $\mu(\omega: \nu) \neq 0$. We choose $U_{1}$, as we may, such that $\mu(\omega: \nu)$ is also analytic on $U_{1}$. Since $c_{P \mid P}^{0}(s: \omega: \nu)$ is holomorphic and unitary when $\mu(\omega: \nu)=0$, it is enough to check that $c_{P \mid P}(1: \omega: \nu)$ and $c_{P \mid P}(s: \omega: \nu)$ are bijective at points $\nu \in U_{1}$ where $\mu(\omega: \nu) \neq 0$. However, since

$$
I_{Q\left(\omega, \tau_{P \mid \bar{P}}\right)}=\mu(\omega: \nu) c_{P \mid \bar{P}}(s: \omega: \nu) c_{P \mid P}(s: \omega: \bar{\nu})^{*}
$$

for all $\nu \in a_{C}^{*}$, it is clear that both $c_{P \mid P}(s: \omega: \nu)$ and $c_{P \mid P}(s: \omega: \bar{\nu})^{*}$ are bijective at any point $\nu \in \mathrm{a}_{\mathrm{C}}^{*}$ where both are analytic and $\mu(\omega: \nu)$ is defined. This proves that $c_{P \mid P}^{0}(s: \omega: \nu)$ is bijective for all $\nu \in U_{1}$ and completes the proof of the lemma.

The following theorem gives a sufficient condition for the existence of complementary series.

THEOREM 3.5. Let $U$ be a neighborhood of zero in $\sqrt{-1} a^{*}(s)$ such that, for all $\nu \in U, C(\nu)$ is positive definite. Then $I\left(P, \sigma_{\nu}\right)$ is unitary for all $\nu \in U$.

Proof. It suffices to show that the representation space $\mathscr{B}(P)^{\sim}$ of $I\left(P, \sigma_{v}\right)^{\sim}$ has a positive definite hermitian form defined on it which is invariant under the operators $I\left(P, \sigma_{\nu}\right)^{\sim}(g)$ for all $g \in G$ and $\nu \in U$. 
We shall show first that $C(\nu)$ induces a right $I\left(P, \sigma_{\nu}\right)$-invariant hermitian form on $\mathcal{T}(\bar{P} \mid P)$. We set

$$
\begin{aligned}
H(\nu)(T, S)=\left(C(\nu) \psi_{T}, \psi_{S}\right) \\
\quad=\int_{M / A} \int_{K \times K}\left(C(\nu) \psi_{T}\right)\left(k_{1}: m: k_{2}\right) \bar{\psi}_{S}\left(k_{1}: m: k_{2}\right) d k_{1} d k_{2} d m^{*} \\
\quad=\int_{M / A} \int_{K \times K} \chi_{\nu}(m)\left(C(\nu) \psi_{T}\right)\left(k_{1}: m: k_{2}\right) \bar{\psi}_{S}\left(k_{1}: m: k_{2}\right) \chi_{-\nu}(m) d k_{1} d k_{2} d m^{*} \\
=\left(\chi_{\nu} C(\nu) \psi_{T}, \chi_{-\nu} \psi_{S}\right)
\end{aligned}
$$

for any $T, S \in \mathcal{T}(\bar{P} \mid P)$ and $\nu \in U$. It follows from Lemma 2.1 and the factorization

$$
\chi_{s \nu} c_{P \mid P}^{0}(s: \omega: \nu) \psi=\chi_{-\nu} c_{P \mid P}(s: \omega: \nu) \chi_{-\nu} \cdot \chi_{\nu} c_{P \mid P}(1: \omega: \nu)^{-1} \chi_{-\nu} \cdot \chi_{\nu} \psi
$$

for any $\nu \in \sqrt{-1} a^{*}(s)$ and $\psi \in \mathbb{Q}\left(\omega, \tau_{P \mid \bar{P}}\right)$ that

$$
\chi_{-\nu} C(\nu) \chi_{-\nu}\left(\alpha^{P} * \psi \chi_{\nu}\right)=\alpha^{P} * \chi_{-\nu} C(\nu) \psi
$$

for any $\alpha \in C_{c}^{\infty}(G), \psi \in \mathbb{Q}\left(\omega, \tau_{P \mid \bar{P}}\right)$ and $\nu \in \sqrt{-1} \mathfrak{a}^{*}(s)$. Thus,

$$
\begin{aligned}
\left(\chi_{-\nu} C(\nu) \chi_{-\nu}\left(\alpha^{P} * \chi_{\nu} \psi_{T}\right), \chi_{\nu} \psi_{S}\right) & =\left(\alpha^{P} * \chi_{-\nu} C(\nu) \psi_{T}, \chi_{\nu} \psi_{S}\right) \\
& =\left(\chi_{-\nu} C(\nu) \psi_{T},\left(\alpha^{P}\right)^{\sim} * \chi_{\nu} \psi_{S}\right) .
\end{aligned}
$$

Since $\alpha^{P} * \chi_{\nu} \psi_{T}=\chi_{\nu} \psi_{T I\left(P, \sigma_{v}\right)\left(\alpha^{\prime}\right)}$ and $\left(\alpha^{\sim}\right)^{P} * \chi_{\nu} \psi_{S}=\psi_{S I\left(P, \sigma_{\nu}\right)\left(\alpha^{-}\right)}\left({ }^{(c-")}\right.$ denotes complex conjugate),

$$
H(\nu)\left(T I\left(P, \sigma_{\nu}\right)\left(\alpha^{\prime}\right), S\right)=H(\nu)\left(T, S I\left(P, \sigma_{\nu}\right)\left(\alpha^{-}\right)\right)
$$

for all $T, S \in \mathcal{T}(\bar{P} \mid P)$ and $\alpha_{1} \in C_{c}^{\infty}(G)$.

In particular, choosing an open compact subgroup $K_{0}$ of $G$ such that both $T$ and $S$ are $K_{0}$-invariant and writing $\alpha_{g}$ for the characteristic function of the double coset $K_{0} g K_{0}$ multiplied by one over its Haar measure, we obtain

$$
\begin{aligned}
H(\nu)\left(T I\left(P, \sigma_{\nu}\right)\left(g^{-1}\right), S\right) & =H(\nu)\left(T I\left(P, \sigma_{\nu}\right)\left(\alpha_{g}^{\prime}\right), S\right) \\
& =H(\nu)\left(T, S I\left(P, \sigma_{\nu}\right)\left(\alpha_{g}\right)\right) \\
& =H(\nu)\left(T, S I\left(P, \sigma_{\nu}\right)(g)\right)
\end{aligned}
$$

for any $g \in G$. Thus, we have shown that $H(\nu)$ is a positive definite hermitian form on $\mathcal{T}(\bar{P} \mid P)$ which is invariant under the operators $I\left(P, \sigma_{\nu}\right)(g)(g \in G)$ acting on the right.

Now fix a nonzero element $h_{0} \in \mathfrak{B}(P)$. Set

$$
\left(h_{1} \tilde{}, h_{2} \tilde{\nu}\right)_{\nu, h_{0}}=H(\nu)\left(h_{0} \otimes h_{1} \tilde{,} h_{0} \otimes h_{2} \tilde{)}\right)
$$

for all $h_{1}^{\tilde{1}}, h_{2}^{\tilde{E} \in \mathscr{B}(P)}$. Since $H(\nu)$ is hermitian and positive definite on $\oiint(P) \otimes \oiint(P)^{\sim},(,)_{\nu, h_{0}}$ is hermitian and positive definite on $\mathscr{B}(P)^{\sim}$. Since

$$
\begin{aligned}
\left(h_{0} \otimes h^{\sim}\right)\left(I\left(P, \sigma_{v}\right)\left(g^{-1}\right) h\right) & =\left\langle h^{\sim}, I\left(P, \sigma_{v}\right)\left(g^{-1}\right) h\right\rangle h_{0} \\
& =\left\langle I\left(P, \sigma_{\nu}\right)^{\sim}(g) h^{\sim}, h\right\rangle h_{0}
\end{aligned}
$$


for all $h \in \mathfrak{B}(P), h^{\sim} \in \mathscr{G}(P)^{\sim}$ and $g \in G$,

$$
\left(I\left(P, \sigma_{v}\right)^{\sim}(g) h_{1}^{\sim}, I\left(P, \sigma_{v}\right)^{\sim}(g) h_{2}^{\sim}\right)_{\nu, h_{0}}=\left(h_{1}^{\sim}, h_{2}^{\sim}\right)_{v, h_{0}}
$$

for all $h_{1}^{\tilde{1}}, \tilde{h_{2}} \in \mathfrak{G}_{(P)^{\sim}}$ and $g \in G$. Thus, $I\left(P, \sigma_{\nu}\right)^{\sim}$ is unitary and, therefore, so is $I\left(P, \sigma_{\nu}\right)$.

\section{REFERENCES}

1. I. M. Gelfand and M. I. Graev, Representations of the group of second-order matrices with elements in a locally compact field and special functions on locally compact fields, Uspehi Mat. Nauk 18 (1963), no. 4 (112), 29-99.

2a. Harish-Chandra, On the theory of the Eisenstein integral (Proc. Conf. Harmonic Analysis, Univ. of Maryland, College Park, Md., 1971), Lecture Notes in Math., vol. 266, Springer-Verlag, Berlin and New York, 1971.

2b. _ Harmonic analysis on reductive p-adic groups, Proc. Sympos. Pure Math., vol. 26, Amer. Math. Soc., Providence, R. I., 1974, pp. 167-192.

3. A. W. Knapp and E. M. Stein, Singular integrals and the principal series. IV, Proc. Nat. Acad. Sci. U.S.A. 72 (1975), 2459-2461.

4. R. G. Laha, Complementary series representations of $\mathrm{GL}(2, D)$, where $D$ is a central division algebra over a non-archimedean local field, Notices Amer. Math. Soc. 25 (1978), p. A-262; Abstract \# 753-B44.

5. G. I. Ol'sanskii, Intertwining operators and complementary series in the class of representations induced from parabolic subgroups of the general linear group over a locally compact division algebra, Mat. Sb. 93 (135), 1974 = Math. USSR Sb. 22 (1974), 217-355.

6. A. J. Silberger, Introduction to harmonic analysis on reductive p-adic groups (based on lectures by Harish-Chandra at the Institute for Advanced Study, Princeton, N. J., 1971-73), Math. Notes, no. 23, Princeton Univ. Press, Princeton, N. J., 1979.

Department of Mathematics, Cleveland State University, Cleveland, Ohio 44115 